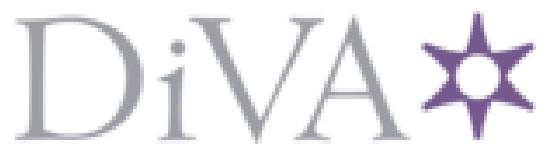

http://www.diva-portal.org

This is the published version of a paper presented at 9th European Conference on Mobile Robots (ECMR 2019), Prague, Czech Republic, September 4-6, 2019.

Citation for the original published paper:

Mielle, M., Magnusson, M., Lilienthal, A. (2019)

A comparative analysis of radar and lidar sensing for localization and mapping

In: 2019 European Conference on Mobile Robots (ECMR) IEEE

N.B. When citing this work, cite the original published paper.

Permanent link to this version:

http://urn.kb.se/resolve?urn=urn:nbn:se:oru:diva-76976 


\title{
A comparative analysis of radar and lidar sensing for localization and mapping
}

\author{
Malcolm Mielle, Martin Magnusson, Achim J. Lilienthal
}

\begin{abstract}
Lidars and cameras are the sensors most commonly used for Simultaneous Localization And Mapping (SLAM). However, they are not effective in certain scenarios, e.g. when fire and smoke are present in the environment. While radars are much less affected by such conditions, radar and lidar have rarely been compared in terms of the achievable SLAM accuracy. We present a principled comparison of the accuracy of a novel radar sensor against that of a Velodyne lidar, for localization and mapping.

We evaluate the performance of both sensors by calculating the displacement in position and orientation relative to a ground-truth reference positioning system, over three experiments in an indoor lab environment. We use two different SLAM algorithms and found that the mean displacement in position when using the radar sensor was less than $0.037 \mathrm{~m}$, compared to $0.011 \mathrm{~m}$ for the lidar. We show that while producing slightly less accurate maps than a lidar, the radar can accurately perform SLAM and build a map of the environment, even including details such as corners and small walls.
\end{abstract}

\section{INTRODUCTION}

Emergency scenarios are dangerous situations where replacing humans by robots can help save lives. Emergency scenarios encompass multiple types of scenarios such as responses after earthquakes, hurricanes, or other natural disasters, and firemen operations. In all cases, hazards such as fires, radiations, or falling structures, create danger for both victims and first responders. Having robot allies can reduce exploration time and help in collecting and processing data, leading to better awareness of the situation and a reduced number of human casualties.

However, robots will need to perform tasks such as $\mathrm{Si}$ multaneous Localization And Mapping (SLAM) in the harsh conditions associated with emergency situations. While the most common sensors used to perform SLAM are lidars, conditions such as smoke, dust, or fire, can corrupt their measurements since laser beams can not go through smoke or dust. On the other hand, radars are unaffected by smoke which make them great candidates for our use case. However, they typically have lower accuracy than lidars and additional noise, e.g. noise induced by multi-bounce reflections. In our work, we are looking at the performance of a novel radar sensor used for SLAM in indoor environments. While using radars would enable robots to perform in situations with smoke, to the best of our knowledge, no principled evaluation of their ability to perform SLAM exists.

Center of Applied Autonomous Sensor Systems (AASS), Örebro University, Sweden. firstname. lastnamedoru.se

This work was funded in part by the EIT Raw Materials project FIREMII under contract number 18011 and by the Swedish Knowledge Foundation under contract number 20140220 (AIR).

978-1-7281-3605-9/19/\$31.00@2019 IEEE
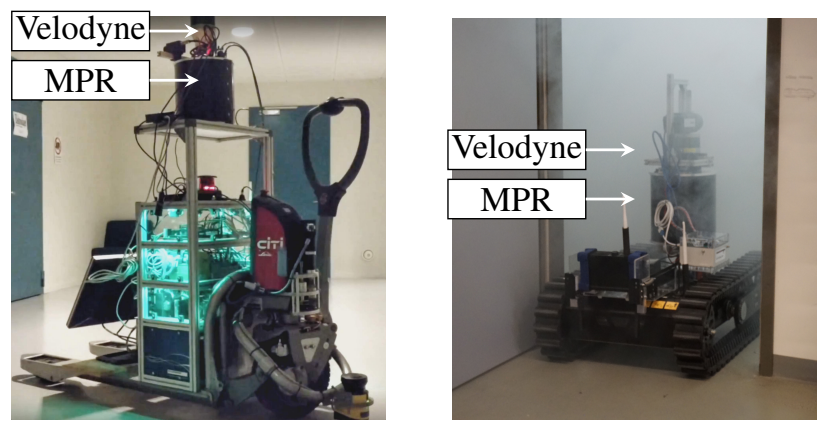

(a) A Linde CiTi forklift platform (b) The Taurob tracker using the with the Velodyne and MPR on top. Velodyne and MPR in smoke.

Fig. 1: Two robot platforms using both the Mechanically Pivotal Radar (MPR) and a Velodyne VLP-16 to take measurements. The MPR is the black cylinder and the Velodyne is mounted on top of the MPR.

\section{CONTRIBUtions}

We present a comparison of the accuracy of a novel radar sensor, the Mechanically Pivoting Radar (MPR) [1], against a Velodyne VLP-16. Both sensors can be seen in Fig. 1a on the robot platform used to conduct the tests presented in our work and performing in smoke in Fig. 1b,

We perform SLAM over three runs in an indoor environment and use two different SLAM algorithms: normal distribution transform occupancy map (NDT-OM) fuser [2], and GMapping [3]. We evaluate the trajectories against ground truth measurements given by a positioning system, and, for each trajectory, evaluate the distance between the final robot pose and the final ground truth pose. We also compare the displacement in position and orientation between each successive SLAM poses and corresponding ground truth measurements. The displacement is calculated using the Relative Pose Error (RPE) derived from the method presented for the KITTI dataset [4]. Throughout the study, we use the same sensor model for both sensors thus demonstrating the ease of use of the radar sensor: performing SLAM can be done using a method originally developed for lidars, and using the radar in place of the lidar.

In summary, we answer the question: what is the accuracy of SLAM using a radar sensor compared to the performance of a lidar, when using the same sensor model for both sensors?

\section{RELATED WORKS}

While no principled evaluation of the performance of radar SLAM compared to lidar SLAM has yet been published, radar sensors have previously been used for SLAM. 
To compare the performances of lidars with radar sensors in adversarial conditions, Ryde and Hillier [5] directed a study using two lidars and a radar in an environment with controlled rain, mist, and dust conditions. They state that neither sensor alone was sufficient for the adverse environments tested but suggest that using both sensor modalities through sensor fusion could enable better mapping.

Chandran and Newman [6] used radar to estimate both the radar map and the vehicle trajectory by maximizing the quality of the map as a function of a motion parametrization. Rouveure et al. [7] present an application in simultaneous localization and mapping using a microwave radar, with a lower resolution than optic cameras or lidar. They perform SLAM by doing a 3D cross-correlation between the current radar image and the constructed map instead of tracking landmarks. Marck et al. [8] and Vivet et al. [9] used radar range measurements to perform SLAM in indoor and outdoor environments respectively. They do not present an evaluation of the accuracy of the SLAM using the radar compared to other sensors, as in our work.

Deissler and Thielecke [10] use a Bat-type UWB radar and Clark and Dissanayake [11] millimiter wave radar to perform SLAM using an EKF and a map of features. The mapping performance of the radar sensors were not compared to an equivalent system using a lidar. Furthermore, they use custom algorithms taking advantage of the radar system to find features of the environment. As such, their radar system can not replace a lidar as is.

Schikora and Romba [12] and Callmer et al. [13] use SIFT features with radar scans. Schikora and Romba [12] present an algorithm for fusion of images from multiple cameras and multiple radars, while Callmer et al. [13] use the long range of the radar to estimate the movement of a vessel and perform loop closing after long travel in open water. Since our radar behaves like a low resolution lidar, extracting features on the scan is not trivial and we instead use SLAM methods based on the registration of successive scans.

Castro and Peynot [14] use lidar and radar to measure smoke or dust concentration by calculating the consistency between the lidar and radar measurements. There is no sensor fusion and the classification depends on a correct calibration of the system. Contrary to our work, they only use the radar to classify lidar measurements as consistent or inconsistent and do not perform SLAM using radar measurements.

Fritsche et al. [15] fuse radar measurements from the MPR and lidar scans and feed them to a SLAM algorithm generating a map of the environment. They show that using the radar and the lidar scans together is beneficial in situations with smoke but perform no general evaluation of the radar performance compared to the lidar.

\section{EXPERIMENT}

The Mechanical Pivoting Radar (MPR) used in our work was developed at Fraunhofer FHR ${ }^{1}$ It is a 2D millimeter wave radar mounted on a pivotal motor, with a rotation speed

${ }^{1}$ https://www.fhr.fraunhofer.de/en.html of $2.5 \mathrm{~Hz}$, and a measurement accuracy of $\pm 3.75 \mathrm{~cm}$ up to $19 \mathrm{~m}$ of range. For every single turn, the MPR returns 200 range measurements, i.e. a measurement every $1.8^{\circ}$. After each full rotation of the radar antenna, the 200 range measurements are merged into one 2D scan of the environment. Since the design of the MPR is simple, the conclusions of our evaluation should be easy to generalize to a wide class of other radars. For more details about the MPR, we refer the reader to the publication by Nowok et al. [1].

For comparison, we used a Velodyne VLP-16 which is a $3 \mathrm{D}$ range scanner with 100-meters range, a channel distribution of $2.00^{\circ}$ between channels, and a range accuracy of $\pm 3 \mathrm{~cm}$. Since the MPR returns 2D point clouds, we convert the $3 \mathrm{D}$ point clouds of the Velodyne to $2 \mathrm{D}$ by slicing them at the MPR's height. To facilitate a fair comparison, each SLAM algorithm produces 2D maps for both the MPR or Velodyne point clouds.

Although the sensor models of the Velodyne and the MPR are different, we use the same sensor model for both the MPR and Velodyne to evaluate the accuracy of each SLAM algorithm when using the MPR in place of the lidar. Indeed, previous work [15] fused the MPR's measurements with the Velodyne's without characterizing the MPR. Using the same sensor model also enable us to show the ease of use of the MPR. Furthermore, taking into account that future work will focus on developing radar-specific sensor and noise models, we present the framework that will be used to evaluate the accuracy of the MPR with an adapted sensor model compared to a Velodyne.

We present the SLAM algorithms we used in Section IVA. the evaluation method in Section IV-B and the experimental setup in Section IV-C

\section{A. SLAM algorithms}

The evaluation is conducted using two state of the art SLAM algorithms: the normal distribution transform occupancy maps (NDT-OM) fuser [2], [16 $]^{2}$ and Gmapping [3 NDT-OM is a grid-based representation where each cell stores a Gaussian distribution representing the shape of the local surface. NDT-OM fuser builds an NDT-OM incrementally by representing each scan as an NDT-OM grid, then registering and fusing it with the map [2]. NDT-OM allows for efficient scan registration [17], [18], planning [19], and localization in both $2 \mathrm{D}$ and 3D. However, pose errors are not corrected at loop closure; thus, errors will add up with time. On the other hand, Gmapping represents the map as an occupancy map and performs localization based on a RaoBackwellized particle filter where each particle carries an individual map of the environment.

The resolution of NDT-OM maps needs to be chosen on a per map basis. In our work, we use a map resolution of $0.75 \mathrm{~m}$ per cell so that the radar can provide enough measurements per cell to approximate the Gaussians. However, it should be noted that the localization and mapping accuracy

2 https://github.com/OrebroUniversity/perception_oru/tree/port-kinetic 3 https://www.openslam.org/ 


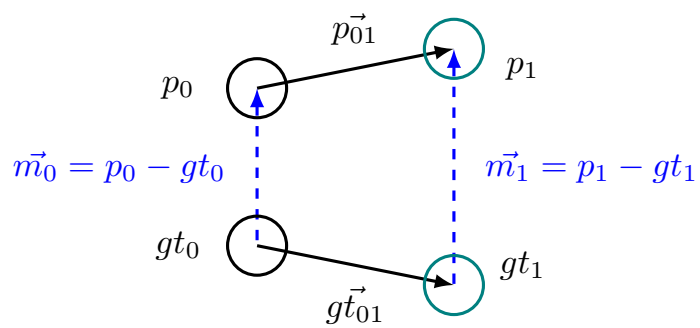

Fig. 2: $p_{0}$ and $p_{1}$ are two SLAM poses while $g t_{0}$ and $g t_{1}$ are the ground truth poses equivalent to $p_{0}$ and $p_{1}$. The displacement in translation is given by calculating $\vec{d}_{t}\left(p_{o}, p_{1}\right)=\overrightarrow{p_{01}} \ominus g \overrightarrow{t_{01}}=$ $\overrightarrow{m_{1}}-\vec{m}_{0}$

is not very sensitive to this parameter as shown by Saarinen et al. [20]. On the other hand, Gmapping has multiple user parameters and we use the default ones given with the ros implementation ${ }^{4}$, In the experiments, both sensors have an uncertainty of $0.1 \mathrm{~m}$ on every beam.

\section{B. Evaluation method}

We evaluate the accuracy of SLAM using the RPE method presented by Geiger et al. [4] for the KITTI dataset. Their method is extended from Kümmerle et al. [21] where the displacement at a pose $i$ is measured against a neighborhood of $n$ poses around it. For each pose $p_{i}$ in the trajectory, with $g t_{i}$ the equivalent pose in the ground truth trajectory, the displacement is expressed as:

$$
d_{s}\left(p_{i}, g t_{i}\right)=\mathrm{R}\left(p_{i}, p_{i+1}\right) \ominus \mathrm{R}\left(g t_{i}, g t_{i+1}\right)
$$

where $\mathrm{R}$ represents the transformation we want to evaluate and $\ominus$ is the invert operator of the standard motion composition operator [21]. In our work, we consider the translation and rotation errors separately, as is done in the work of Geiger et al. [4]. The calculation of the displacement in translation is illustrated in Fig. 2 .

In Table I. we evaluate six types of relations between the SLAM and ground truth trajectories. The first four rows correspond to the errors between the final pose of the robot and the equivalent ground truth pose. We evaluate the Euclidean distance $D_{e}$, the distance along the $\vec{x}$ and $\vec{y}$ axis ( $D_{e_{\vec{x}}}$ and $D_{e_{\vec{y}}}$ respectively), and the angle difference $D_{o}$. The last two rows correspond to the mean error along the trajectory. We look at $d_{e}$ and $d_{o}$ the mean of the norms of the displacement in translation and rotation between each successive pose. While displacement measures give us an evaluation of the local consistency of the trajectory, the error at the end pose enables us to judge the quality of the overall trajectory. We can express $\vec{d}_{t}\left(\vec{p}_{i}, g \vec{t}_{i}\right)$ the vector of the displacement along $\vec{x}$ and $\vec{y}$ as:

$$
\vec{d}_{t}\left(\vec{p}_{i}, g \vec{t}_{i}\right)=\left(\vec{p}_{i+1}-\vec{p}_{i}\right)-\left(g \vec{t}_{i+1}-g \vec{t}_{i}\right)
$$

with $\vec{p}_{i}$ and $g \vec{t}_{i}$ the coordinate vectors of the robot and the equivalent ground truth pose in the map coordinate frame. Similarly, we express the displacement in orientation $d_{t}\left(o_{i}, g o_{i}\right)$ as the difference between the smallest signed angle between $o_{i}$ and $o_{i+1}$, and the smallest signed angle

${ }^{4}$ https://wiki.ros.org/gmapping

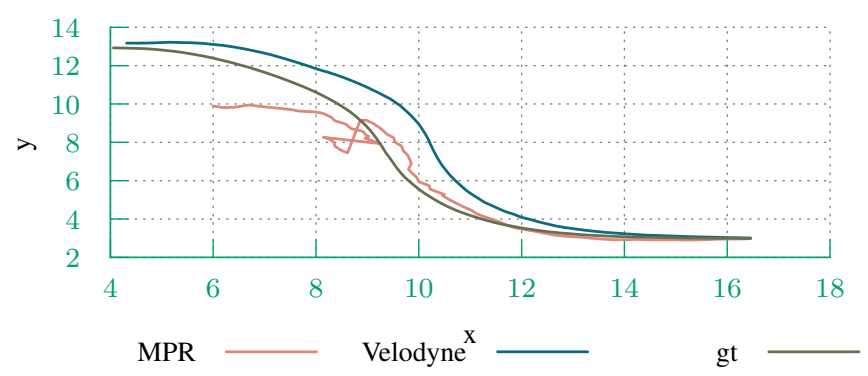

Fig. 3: Trajectories of the robot using both sensor modalities and NDT-OM fuser for the first run. The large error in the MPR trajectory is due to two erroneous registrations.

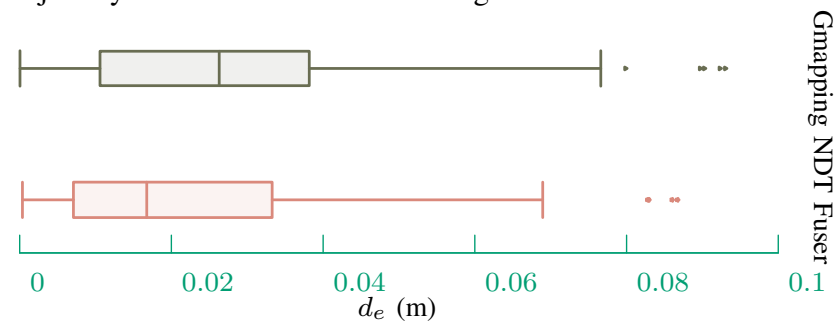

Fig. 4: This figure shows a boxplot of the absolute displacement in position $d_{e}$ using the MPR for the first run. One can see that results are similar for NDT Fuser and Gmapping.

between $g o_{i}$ and $g o_{i+1}$. Hence $d_{t}\left(o_{i}, g o_{i}\right)$ is bounded between $\pi$ and $-\pi$.

\section{Experimental setup}

We used a self-driving CiTi forklift platform developed by Linde and mounted the MPR and Velodyne on top of the machine, as seen in Fig. 1a We recorded three datasets at Örebro University and the resulting maps can be seen in Fig. 7 and Fig. 8. In the first dataset, the robot went from the top of the map toward the bigger room at the bottom. In the second dataset, the robot started at the bottom of the map and moved toward the top. In the third dataset, the robot started from the top, went to the bottom, and returned to its initial position after performing a $360^{\circ}$ rotation. While the first and second datasets do not have people walking in the environment, the third dataset has few people walking close to the robot during its rotation. The choice of the environment was mainly conditioned by the availability of a ground truth localization system, enabling us to compare the SLAM trajectories against the actual position of the forklift. The ground truth positioning system is a commercial system for indoor positioning by Kollmorgen and uses retroreflective markers. The system has a positional error $<1 \mathrm{~cm}$ and an error in orientation $<0.005 \mathrm{rad}$. Finding correspondences between SLAM and ground-truth poses is done by comparing their time-stamps. Recordings of the sensor measurements are available as ROS bag files online [22].

\section{RESUlts}

The evaluation measures described in Section IV-B are presented for each dataset individually in Table I and summarized in Table $\amalg$ 


\begin{tabular}{|c|c|c|c|c|c|c|c|c|c|c|c|c|}
\hline & \multicolumn{4}{|c|}{ First run } & \multicolumn{4}{|c|}{ Second Run } & \multicolumn{4}{|c|}{ Third Run } \\
\hline & \multicolumn{2}{|c|}{ NDT fuser } & \multicolumn{2}{|c|}{ Gmapping } & \multicolumn{2}{|c|}{ NDT fuser } & \multicolumn{2}{|c|}{ Gmapping } & \multicolumn{2}{|c|}{ NDT fuser } & \multicolumn{2}{|c|}{ Gmapping } \\
\hline & MPR & Velodyne & MPR & Velodyne & MPR & Velodyne & MPR & Velodyne & MPR & Velodyne & MPR & Velodyne \\
\hline$D_{e}(\mathrm{~m})$ & 2.723 & 0.301 & 1.450 & 0.271 & 1.201 & 0.375 & 0.744 & 0.384 & 0.171 & 0.084 & 0.042 & 0.043 \\
\hline$D_{e_{\vec{x}}}(\mathrm{~m})$ & -1.515 & 0.192 & 1.417 & 0.147 & 0.672 & -0.226 & -0.704 & -0.220 & -0.169 & 0.006 & -0.018 & 0.001 \\
\hline$D_{e_{\vec{y}}}(\mathrm{~m})$ & -2.262 & 0.233 & 0.310 & 0.228 & 0.995 & -0.297 & -0.239 & -0.313 & -0.017 & -0.083 & -0.036 & -0.042 \\
\hline$D_{o}(\mathrm{rad})$ & 0.663 & -0.005 & 0.362 & -0.023 & 0.250 & 0.002 & 0.069 & 0.005 & -0.039 & 0.005 & 0.022 & -0.013 \\
\hline \multirow{2}{*}{$d_{e}(\mathrm{~m})$} & 0.023 & 0.004 & 0.029 & 0.009 & 0.031 & 0.004 & 0.027 & 0.011 & 0.029 & 0.004 & 0.037 & 0.010 \\
\hline & \pm 0.021 & \pm 0.003 & \pm 0.023 & \pm 0.017 & \pm 0.038 & \pm 0.003 & \pm 0.029 & \pm 0.013 & \pm 0.035 & \pm 0.003 & \pm 0.048 & \pm 0.010 \\
\hline \multirow{2}{*}{$d_{o}(\mathrm{rad})$} & 0.069 & 0.002 & 0.067 & 0.007 & 0.082 & 0.002 & 0.070 & 0.007 & 0.087 & 0.008 & 0.076 & 0.006 \\
\hline & \pm 0.09 & \pm 0.002 & \pm 0.131 & \pm 0.006 & \pm 0.121 & \pm 0.002 & \pm 0.159 & \pm 0.007 & \pm 0.163 & \pm 0.068 & \pm 0.159 & \pm 0.006 \\
\hline
\end{tabular}

TABLE I: The distances to the final ground poses for each run and the mean displacements \pm one standard deviation for each run. The distances to the ground truth and displacements in position are given in meters while the displacements in orientation and angles between the final SLAM poses and ground truth poses are given in radians. NDT and Gmapping have similar displacements in position and orientation. the mean displacements for both SLAM algorithms stayed under $0.037 \mathrm{~m}$ and $0.087 \mathrm{rad}$.

\begin{tabular}{lrrrrr}
\hline & \multicolumn{2}{c}{ NDT } & & \multicolumn{2}{c}{ Gmapping } \\
\cline { 2 - 3 } \cline { 5 - 6 } & \multicolumn{1}{c}{ MPR } & Velodyne & & MPR & Velodyne \\
\hline \multirow{2}{*}{$D_{e}(\mathrm{~m})$} & 1.365 & 0.254 & & 0.746 & 0.233 \\
& \pm 1.049 & \pm 0.575 & & \pm 0.124 & \pm 0.142 \\
$D_{e_{\vec{x}}}(\mathrm{~m})$ & -0.337 & 0.232 & & -0.009 & -0.024 \\
$D_{e_{\vec{y}}}(\mathrm{~m})$ & \pm 0.901 & \pm 0.884 & & \pm 0.171 & \pm 0.151 \\
$D_{o}(\mathrm{rad})$ & -0.427 & 0.012 & & -0.048 & -0.042 \\
& \pm 1.362 & \pm 0.227 & & \pm 0.218 & \pm 0.221 \\
& 0.292 & 0.001 & & 0.151 & -0.010 \\
\multirow{2}{*}{$d_{e}(\mathrm{~m})$} & \pm 0.289 & \pm 0.151 & & \pm 0.005 & \pm 0.012 \\
& 0.028 & 0.004 & & 0.031 & 0.010 \\
$d_{o}(\mathrm{rad})$ & \pm 0.004 & \pm 0.005 & & \pm 0.005 & \pm 0.001 \\
& 0.080 & 0.004 & & 0.072 & 0.007 \\
& \pm 0.008 & \pm 0.004 & & \pm 0.003 & \pm 0.001 \\
\hline
\end{tabular}

TABLE II: This table presents the mean \pm one standard deviation over all run of the results presented in Table 1 per SLAM algorithm and sensor modality.

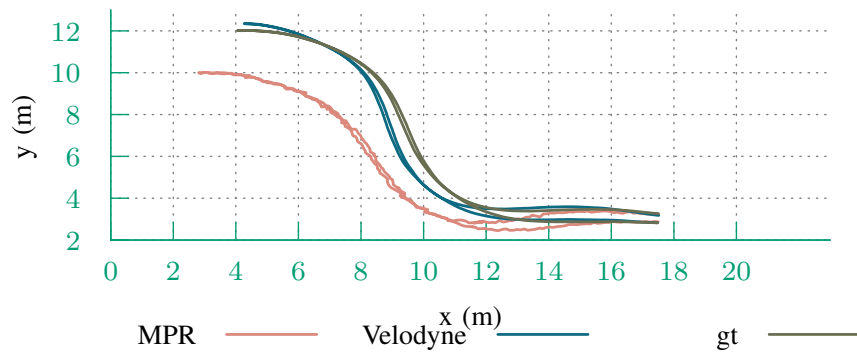

Fig. 5: Trajectories of the robot using both sensor modalities and NDT-OM fuser for the third run. A small rotation error at the beginning shifted the MPR's trajectory. However, the rest of the trajectory is correct as shown by the low displacement in position of the MPR at each point.

Looking at Table III. Gmapping estimates trajectories closer to the ground truth end poses than NDT-OM fuser, when using the MPR, while NDT-OM fuser performs slightly better when using the Velodyne. However, the detailed results in Table II show that the lower performance of NDT-OM fuser when using the MPR is mainly due to the first dataset. Indeed, the first dataset's trajectory using NDT-OM fuser and the MPR does not follow the ground truth closely. However, the differences between both trajectories are due to isolated erroneous registrations, as visible in Fig. 3. On the other hand, the mean of the norm in displacement in translation $d_{e}$ and orientation $d_{o}$ of NDT-OM fuser and Gmapping are not significantly different, as shown in Fig. 4. The fact that

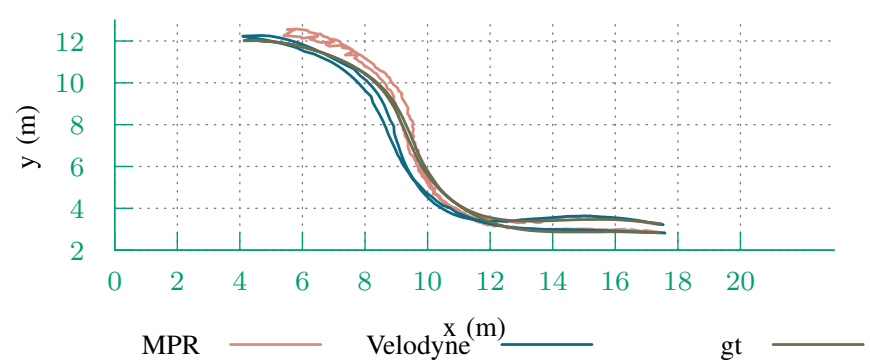

Fig. 6: Trajectories of the robot using Gmapping and both sensor modalities for the third run. The MPR and lidar's trajectories are similar.

$d_{e}$ and $d_{o}$ are similar for both SLAM algorithms shows that they overall performed similarly and both Gmapping and NDT-OM fuser produce trajectories with similar amounts of distortion. Indeed, the maximum displacement in translation for each dataset is similar for both NDT-OM fuser and Gmapping: apart from some outliers, the displacement in translation between both SLAM algorithms is under $0.1 \mathrm{~m}$.

Since Gmapping usually estimates the trajectory slightly closer to the ground-truth than NDT-OM fuser, but has larger displacements $d_{e}$ and $d_{o}$, the displacement is mainly caused by errors and corrections along the trajectory. On the other hand, NDT-OM fuser trajectories using the MPR are farther away from the ground truth than Gmapping, as visible in Fig. 3. Fig. 5, and Fig. 6. Since the mean displacement in position $d_{e}$ of NDT-OM fuser is lower than Gmapping's, we can deduce that the differences between its trajectories and the ground truth's trajectories are due to small rotation offsets over the trajectories. An example of the effect of such errors in rotation is visible as a slight bending of the top corridor in Fig. 7e. Hence, while Gmapping's trajectories follow the ground truth more accurately than NDT-OM fuser, the trajectories estimated by NDT-OM fuser are smoother with fewer jumps in position than Gmapping.

Looking at the NDT-OM fuser maps in Fig. 7 and GMapping maps in Fig. 8, one can see that all maps, whether they were built using the MPR or the Velodyne, represent the environment with details, such as corners and straight walls. Since the trajectories estimated with the Velodyne are closer to the ground truth than with the MPR, we can assume that the maps built with the Velodyne are more accurate. 


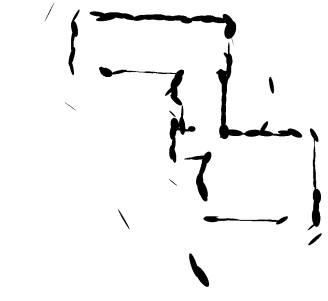

(a) NDT-OM fuser map built using the MPR on the first dataset.

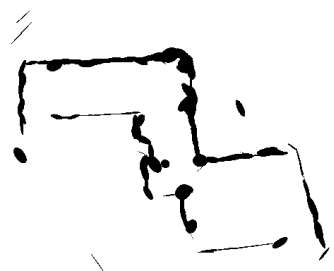

I

(c) NDT-OM fuser map built using the MPR on the second dataset.

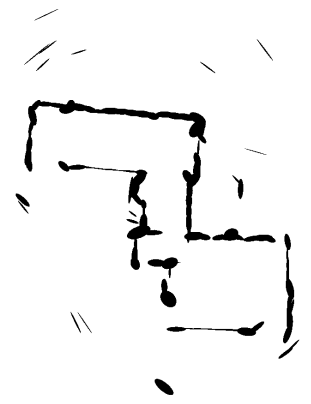

(e) NDT-OM fuser map built using the MPR on the third dataset.

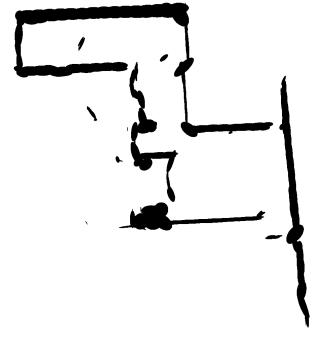

(b) NDT-OM fuser map built using the Velodyne on the first dataset.

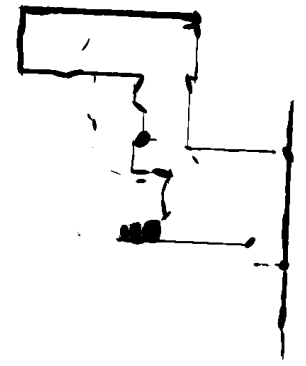

(d) NDT-OM fuser map built using the Velodyne on the second dataset.

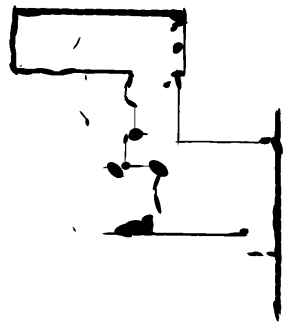

(f) NDT-OM fuser map built using the Velodyne on the third dataset.
Fig. 7: Maps built using the MPR and the Velodyne with NDT-OM fuser as a SLAM algorithm. Even with errors such as bent corridors, the environment is recognizable in all maps and the robot was able to localize in them.

By comparing the MPR maps to the Velodyne maps, one can see that, when using the MPR, both Gmapping and NDT maps have a slight misalignment between corridors. Furthermore, NDT-OM fuser maps can have local scaling errors: the middle corridor of Fig. $7 \mathrm{c}$ is smaller than its actual size due to bad MPR scan registrations.

\section{DISCUSSION AND FUTURE WORK}

Given the low resolution of the MPR, our results are highly encouraging. Independently of which SLAM algorithm was used, it was possible to build detailed maps of the environment with the MPR, (see Fig. 7) and Fig. 8). Furthermore, the robot was able to localize itself in the maps when using the MPR, effectively performing SLAM. However, both SLAM algorithms were more prone to mapping errors when using the MPR, due its lower resolution.

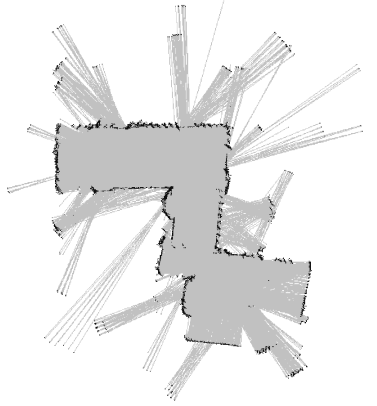

(a) Gmapping map built using the MPR on the first dataset.

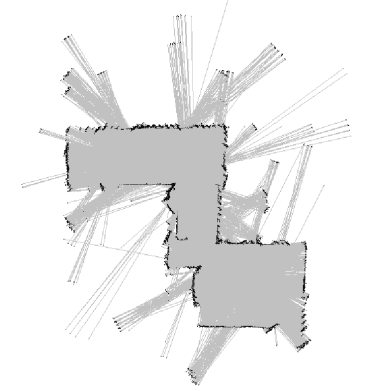

(c) Gmapping map built using the MPR on the second dataset.

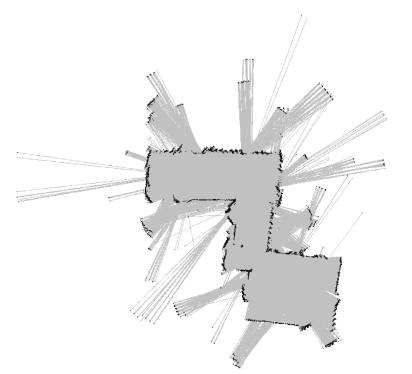

(e) Gmapping map built using the MPR on the third dataset.

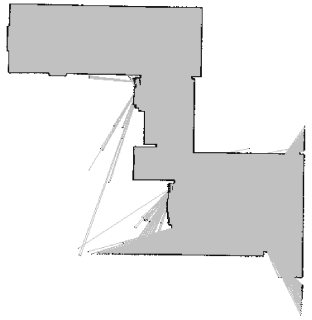

(b) Gmapping map built using the Velodyne on the first dataset.

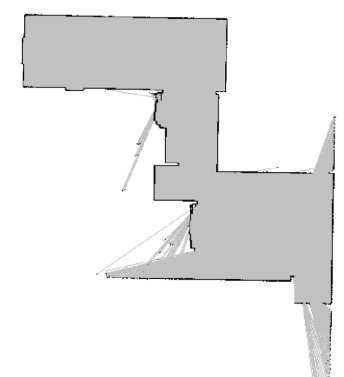

(d) Gmapping map built using the Velodyne on the second dataset.

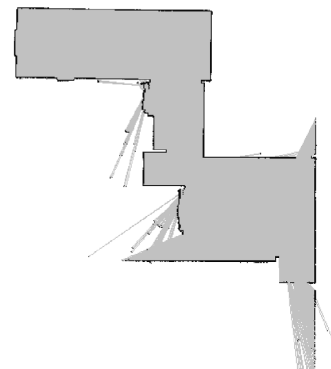

(f) Gmapping map built using the Velodyne on the third dataset.
Fig. 8: Maps built using the MPR and the Velodyne with Gmapping as a SLAM algorithm. Again, the environment is recognizable in all maps and the robot could localize in them.

More specifically, Gmapping estimates trajectories closer to the ground truth than NDT-OM fuser. As seen in the four first rows of Table II Gmapping error between the final pose of the robot and the equivalent ground truth pose is lower than NDT-OM fuser, and, as visible in Fig. 5 and Fig. 6 the trajectories are closer to the ground truth. On the other hand, NDT-OM fuser's displacement in position is slightly lower than that of Gmapping with $d_{e}=0.028 \pm 0.004$ against $0.031 \pm 0.005$. One reason Gmapping estimates trajectories closer to the ground truth could be found in the mapping process: compared to NDT maps, occupancy grids may be less sensitive to noise in the radar measurements due to their lower level of details. On the other hand, NDTOM fuser assumes that the MPR is as accurate as the Velodyne since for the experiments in this paper we have used the same sensor model for both the MPR and the 
Velodyne. Thus, NDT scan registrations are typically more accurate, leading to smoother trajectories with the caveat that erroneous registrations will lead to large isolated errors (as seen in Fig. 3).

Our study shows that the MPR, and possibly similar types of radars, can be used to perform SLAM as is, without the need to change the sensor model from the one used by the lidar. Taking into account that the Velodyne will not be useful in scenarios with smoke or dust, being able to use the MPR instead would be beneficial in such situations.

Future work will be focused on developing radar-specific sensor and noise models to obtain the best performance from the MPR. Furthermore, we will extend the evaluation of the MPR to a scenario with actual smoke.

By showing that we can perform SLAM using the MPR and classic SLAM algorithms, we open the way toward using this technology, and thus robots, in harsh conditions and actual scenarios with smoke or dust in the air.

\section{SUMMARY AND CONCLUSIONS}

We have presented a quantitative comparison of the accuracy of radar versus lidar sensing for indoor localization and mapping. We show that while producing slightly less accurate maps than a lidar, the radar can accurately perform SLAM and build a map of the environment, even including details such as corners and small walls.

We evaluated the radar using two SLAM algorithms: Gmapping and NDT. While Gmapping estimated the overall trajectories closer to the ground truth, NDT fuser had smoother trajectories and slightly lower displacement in position. This can be explained by intrinsic characteristics of the different map types. Both SLAM algorithms obtained similar localization accuracy when using the radar sensor, within around $0.030 \mathrm{~cm}$ and 0.075 radians mean errors. Our results highlight that the radar is a valid alternative to lidar sensing, which is especially important in low-visibility situations.

\section{REFERENCES}

[1] S. Nowok, S. Kueppers, H. Cetinkaya, M. Schroeder, and R. Herschel, "Millimeter wave radar for high resolution 3d near field imaging for robotics and security scans," in Radar Symposium (IRS), IEEE, 2017.

[2] T. Stoyanov, J. Saarinen, H. Andreasson, and A. J. Lilienthal, "Normal distributions transform occupancy map fusion: Simultaneous mapping and tracking in large scale dynamic environments," in IEEE/RSJ International Conference on Intelligent Robots and Systems, IEEE, 2013.

[3] G. Grisetti, C. Stachniss, and W. Burgard, "Improved techniques for grid mapping with rao-blackwellized particle filters," IEEE transactions on Robotics, vol. 23, no. 1, 2007.

[4] A. Geiger, P. Lenz, and R. Urtasun, "Are we ready for autonomous driving? the kitti vision benchmark suite," in 2012 IEEE Conference on Computer Vision and Pattern Recognition, IEEE, 2012, pp. 3354-3361.

[5] J. Ryde and N. Hillier, "Performance of laser and radar ranging devices in adverse environmental conditions," en, Journal of Field Robotics, 2009. DOI: 10.1002/rob.20310.

[6] M. Chandran and P. Newman, "Motion estimation from map quality with millimeter wave radar," in 2006 IEEE/RSJ International Conference on Intelligent Robots and Systems, Oct. 2006. DOI: 10.1109/IROS.2006.281673
[7] R. Rouveure, M. O. Monod, and P. Faure, "High resolution mapping of the environment with a ground-based radar imager," in 2009 International Radar Conference "Surveillance for a Safer World" (RADAR 2009), Oct. 2009, pp. 1-6.

[8] J. W. Marck, A. Mohamoud, E. v. Houwen, and R. v. Heijster, "Indoor radar SLAM a radar application for vision and GPS denied environments," in 2013 European Radar Conference, Oct. 2013.

[9] D. Vivet, F. Gérossier, P. Checchin, L. Trassoudaine, and R. Chapuis, "Mobile ground-based radar sensor for localization and mapping: An evaluation of two approaches," International Journal of Advanced Robotic Systems, vol. 10, no. 8, p. 307, 2013. DOI: $10.5772 / 56636$

[10] T. Deissler and J. Thielecke, "UWB SLAM with raoblackwellized monte carlo data association," in International Conference on Indoor Positioning and Indoor Navigation (IPIN), 2010.

[11] S. Clark and G. Dissanayake, "Simultaneous localisation and map building using millimetre wave radar to extract natural features," in Proceedings 1999 IEEE International Conference on Robotics and Automation (Cat. No.99CH36288C), May 1999. DOI: 10.1109/ROBOT.1999.772543.

[12] M. Schikora and B. Romba, "A framework for multiple radar and multiple 2d/3d camera fusion," in 4th German Workshop Sensor Data Fusion: trends, Solutions, Applications, 2009.

[13] J. Callmer, D. Törnqvist, F. Gustafsson, H. Svensson, and P. Carlbom, "Radar SLAM using visual features," EURASIP Journal on Advances in Signal Processing, vol. 2011, Dec. 1, 2011, ISSN: 1687-6180. DOI: 10.1186/1687-6180-2011-71

[14] M. Castro and T. Peynot, "Laser-to-radar sensing redundancy for resilient perception in adverse environmental conditions," in Proc. of Australasian Conf. on Robotics and Autom., Sydney, Australia, DTIC Document, 2012.

[15] P. Fritsche, B. Zeise, P. Hemme, and B. Wagner, "Fusion of radar, lidar and thermal information for hazard detection in low visibility environments," in 2017 IEEE International Symposium on Safety, Security and Rescue Robotics (SSRR), Oct. 2017, pp. 96-101. DOI: 10.1109/SSRR.2017.8088146

[16] P. Biber and W. Straßer, "The normal distributions transform: A new approach to laser scan matching," in International Conference on Intelligent Robots and Systems, IEEE, 2003.

[17] S. Pang, D. Kent, X. Cai, H. Al-Qassab, D. Morris, and H. Radha, "3d scan registration based localization for autonomous vehicles - a comparison of ndt and icp under realistic conditions," in 2018 IEEE 88th Vehicular Technology Conference (VTC-Fall), Aug. 2018. DOI: 10.1109/VTCFall. 2018.8690819

[18] M. Magnusson, N. Vaskevicius, T. Stoyanov, K. Pathak, and A. Birk, "Beyond points: Evaluating recent 3d scan-matching algorithms," in 2015 IEEE International Conference on Robotics and Automation (ICRA), IEEE, 2015.

[19] T. Stoyanov, M. Magnusson, H. Andreasson, and A. J. Lilienthal, "Path planning in $3 \mathrm{~d}$ environments using the normal distributions transform," in Intelligent Robots and Systems (IROS), IEEE, 2010, pp. 3263-3268.

[20] J. Saarinen, H. Andreasson, T. Stoyanov, and A. J. Lilienthal, "Normal distributions transform monte-carlo localization (NDT-MCL)," in Intelligent Robots and Systems (IROS), 2013 IEEE/RSJ International Conference on, IEEE, 2013, pp. 382-389.

[21] R. Kümmerle, B. Steder, C. Dornhege, M. Ruhnke, G. Grisetti, C. Stachniss, and A. Kleiner, "On measuring the accuracy of SLAM algorithms," Autonomous Robots, Nov. 1, 2009. DOI: $10.1007 / \mathrm{s} 10514-009-9155-6$

[22] M. Mielle, M. Magnusson, and A. J. Lilienthal, "Novel radar datasets," Sep. 2017. DOI: 10.5281/zenodo.893154 